\title{
Disseminated Carcinomatosis of Bone Marrow Due to Sigmoid Colon Cancer
}

\author{
Christos Konstantinidis*, Panagiotis Varsos, Sotirios Kympouris* \\ Surgical Department, Hippocratio General Hospital of Athens, 114 Vasilissis Sofias str, Athens, Region of Attica, Greece \\ *Corresponding author: drchriskons@yahoo.gr
}

Received February 08, 2015; Revised February 24, 2015; Accepted March 03, 2015

\begin{abstract}
Introduction: Disseminated carcinomatosis of the bone marrow (DCBM) caused by colorectal cancer is rarely seen. DCBM is often accompanied with diffuse intravascular coagulation (DIC). Presentation Of Case: A 72-year-old male patient with a history of right nephrectomy three decades ago for referred benign disease, underwent sigmoid colon resection for cancer causing bowel obstruction. The preoperative evaluation revealed elevated values of CA 19-9 and PSA, with no metastatic lesions in abdominal CT. On eighth postoperative day, the patient developed fever with anemia and thrombocytopenia. Bone marrow examination revealed disseminated carcinomatosis caused by colon cancer. The patient deceased on the twenty seventh postoperative day due to the rapid progression of the accompanied disseminated intravascular coagulation. Discussion: Prognosis for patients with cancer who develop disseminated carcinomatosis of bone marrow (DCBM) is poor. Conclusion: In our case, the rapid deterioration of the patient gave us no option for adjuvant chemotherapy. The outcome was fatal. A 72year-old male patient with a history of right nephrectomy for benign disease underwent sigmoid colon resection for bowel obstruction due to cancer. Tumor markers CA 19-9 and PSA were elevated, with no metastatic lesions in abdominal CT of admission. On eighth postoperative day, the patient developed severe anemia and thrombocytopenia. Bone marrow examination revealed disseminated carcinomatosis caused by colon cancer. The patient deceased on the twenty seventh postoperative day due to the rapid progression of the accompanied disseminated intravascular coagulation.
\end{abstract}

Keywords: sigmoid colon, cancer, obstruction, disseminated carcinomatosis, disseminated intravascular coagulation

Cite This Article: Christos Konstantinidis, Panagiotis Varsos, and Sotirios Kympouris, "Disseminated Carcinomatosis of Bone Marrow Due to Sigmoid Colon Cancer." American Journal of Medical Case Reports, vol. 3, no. 4 (2015): 102-104. doi: 10.12691/ajmcr-3-4-4.

\section{Introduction}

Disseminated carcinomatosis of the bone marrow (DCBM) caused by colorectal cancer is rarely seen. In most cases, DCBM is derived from gastric cancer. The prognosis of the disease is poor, especially when it is complicated with disseminated intravascular coagulation (DIC).We report a fatal case of early and rapid manifestation of DCBM accompanied by DIC, which was developed after surgically treatment of sigmoid colon cancer causing bowel obstruction.

\section{Case Report}

A 72-year-old male patient was admitted to our hospital complaining for abdominal discomfort and constipation. He had a medical history of right nephrectomy for cystic lesions. Physical examination showed moderate abdominal distension. Hematological and blood biochemical examinations were normal, while tumor markers CEA, CA19-9 and PSA had elevated values
(13.04 ng/ml, $623.37 \mathrm{U} / \mathrm{ml}$ and $28.71 \mathrm{ng} / \mathrm{ml}$ respectively) (Table 1). Abdominal CT revealed a mass from the wall of the sigmoid colon which was causing obstruction of the lumen (Figure 1, Figure 2). The patient underwent nine days after his admission a scheduled resection of the sigmoid colon, after the placement of a stent at the left ureter, with primary end-to-end anastomosis. The histopathological examination of the specimen revealed middle grade adenocarcinoma (Figure 3), without penetration of the wall of the colon but with extensive lymphatic invasions (positive 10 of 10 lymph nodes and other more lymphatic blocks) (Figure 4). The postoperative period was uneventful until the fifth postoperative day, when the complete blood cell account revealed mild thrombocytopenia ( 100,000/uL), which worsened rapidly the following days. On eighth postoperative day, the patient developed fever with anemia and severe thrombocytopenia. The total amount of platelets dropped to levels less than 20,000/uL. Despite blood infusions the amount of platelets remained at extremely low levels. Additional abdominal CT revealed lesions at the body of lumbar vertebra while chest CT demonstrated diffuse peripheral pulmonary emboli (Figure 5), laboratory test for HITTS were negative for 
pathological findings. A subsequent bone marrow examination revealed diffuse neoplastic infiltration caused by moderate differentiated adenocarcinoma (CK20+, CK7-, CD10-), indicative of disseminated carcinomatosis of the bone marrow from colon cancer (Figure 6, Figure 7). Due to patient's rapid deterioration, treatment with chemotherapy wasn't an option. The patient deceased twenty seven days after initial surgical operation.

Table 1. Laboratory Data on Admission

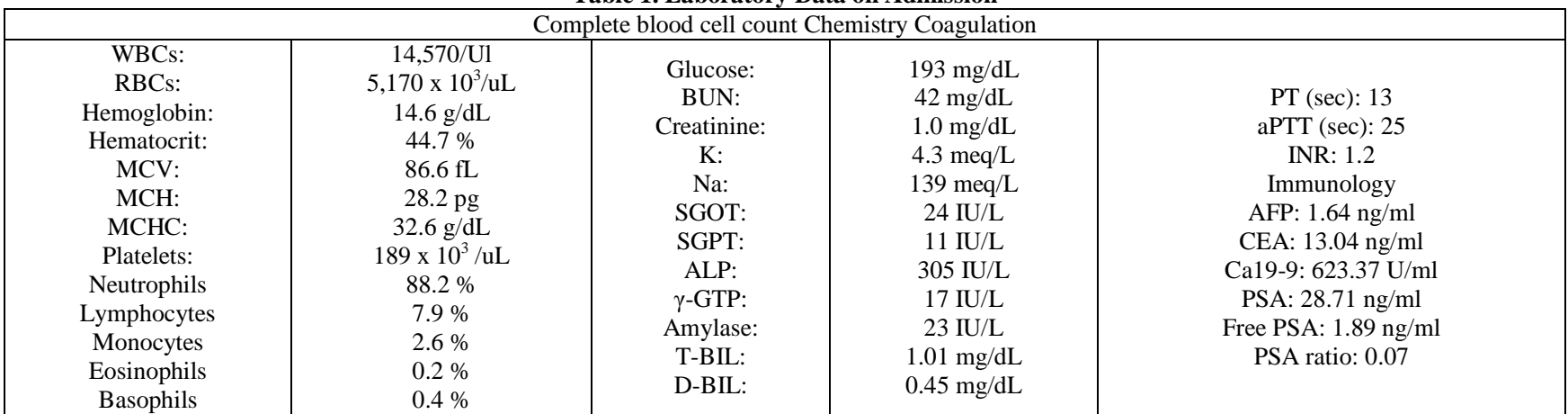

\section{Discussion}

Patients with disseminated carcinomatosis of the bone marrow complain for back pain and bleeding tendencies, as was with our patient, presented as purpura. The peripheral blood examination reveals severe anemia with thrombocytopenia, elevated alkaline phosphatase and lactase dehydrogenase. Bone marrow examination demonstrates a diffusely infiltration rather than a nodular pattern.

A review of the literature shows that most studies related to disseminated carcinomatosis of the bone marrow (DCBM) were conducted in Japan. In these studies, the origin of DCBM is advanced gastric cancer, in up to $90 \%$ of cases. In the contrary, colorectal cancer remains a rare cause of DCBM, with rectal cancer slightly more frequent. In one study, only 27 cases of colorectal cancer are reported, as an origin of DCBM, between 1984 and 2013 in Japan [1].

Prognosis of disseminated carcinomatosis of bone marrow (DCBM) accompanied with diffuse intravascular coagulation (DIC) in patients with cancer is poor. Standard anticoagulant therapy is considered to be ineffective for DIC caused by malignancies [2]. Other treatment options are administration of fresh frozen plasma and platelet replacement, with or without anticoagulation therapy, which also has little impact in patientssurvival, who usually die within 1-3 weeks [3].

Targeting towards the underlying cancer would offer better survival rates. Various chemotherapy regimens are used for this purpose with different outcomes [4-12]. Although survival is improved in most cases, patients with colorectal cancer and DCBM do not survive more than 100 days [1]. Yoshida et al [13] report the early start of chemotherapy after surgery in patients with colorectal cancer and synchronous metastases, aiming at preventing tumor growth. The effect and safety of such an approach needs further confirmation with prospective studies.

Unfortunately in our case, the rapid deterioration of the patient gave us no option for adjuvant chemotherapy with a better outcome. His general condition was severe on the fourteenth postoperative day and thereafter, making any administration of chemotherapy prohibitive. The general condition of the patient didn't improve and he, finally, deceased on the twenty seventh postoperative day.

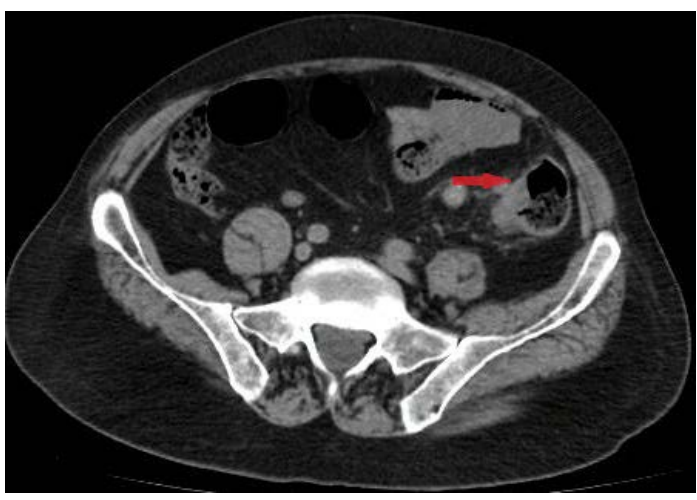

Figure 1. Abdominal CT scans in series indicating the sigmoid colon cancer, obstructing the lumen of the bowel (red arrows).

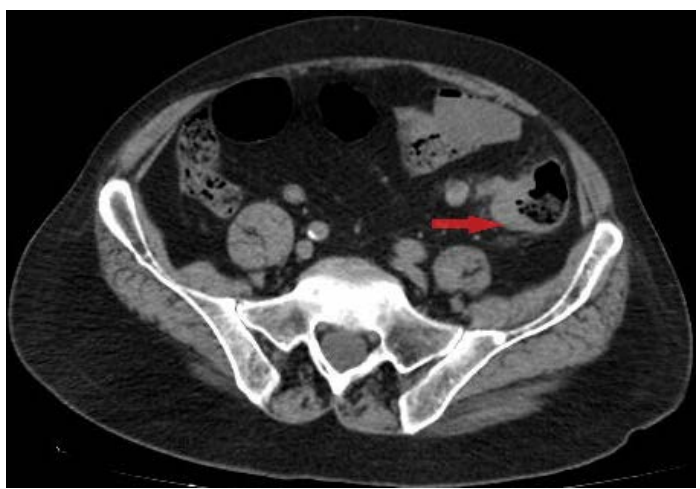

Figure 2. Abdominal CT scans in series indicating the sigmoid colon cancer, obstructing the lumen of the bowel (red arrows).

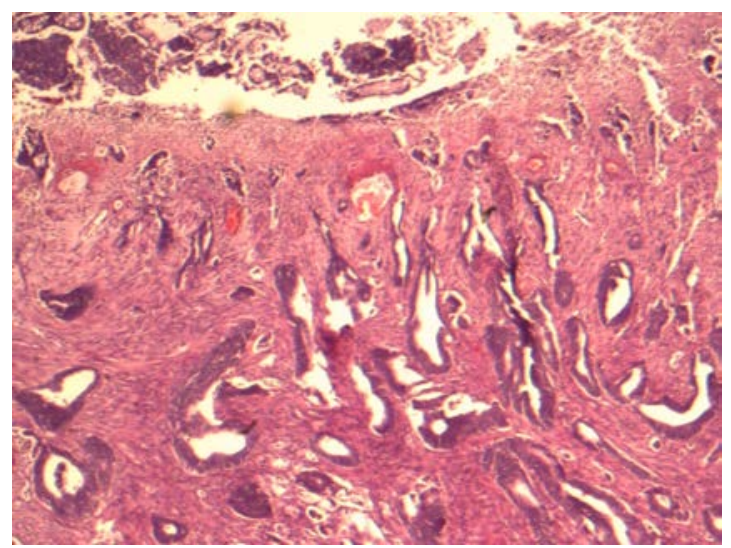

Figure 3. Histopathological specimen examination of the sigmoid colon cancer (H\&E X 25) 


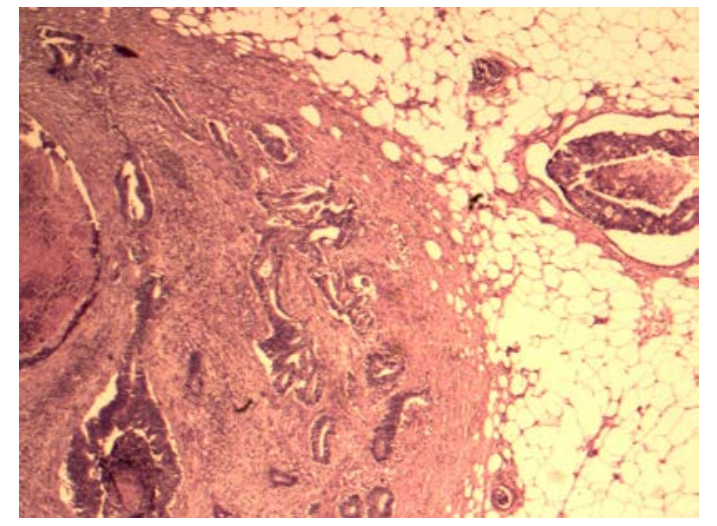

Figure 4. Histopathological examination of infiltrated lymph node from the sigmoid colon cancer ( H\&E X 25)

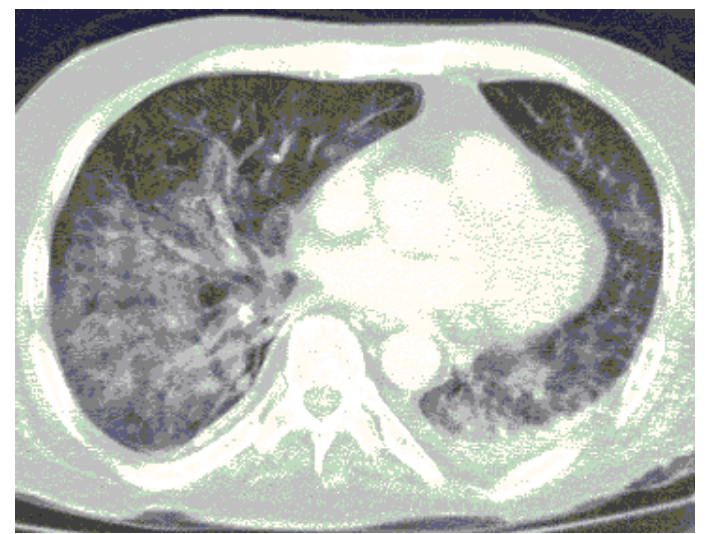

Figure 5. Chest CT scan indicating diffuse peripheral pulmonary emboli.

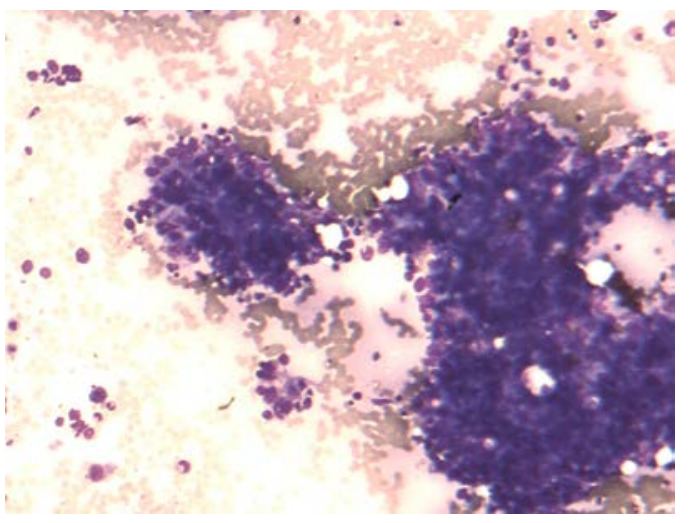

Figure 6. Bone marrow examination of the ilium that reveals a cluster of abnormal cells (stain X 100)

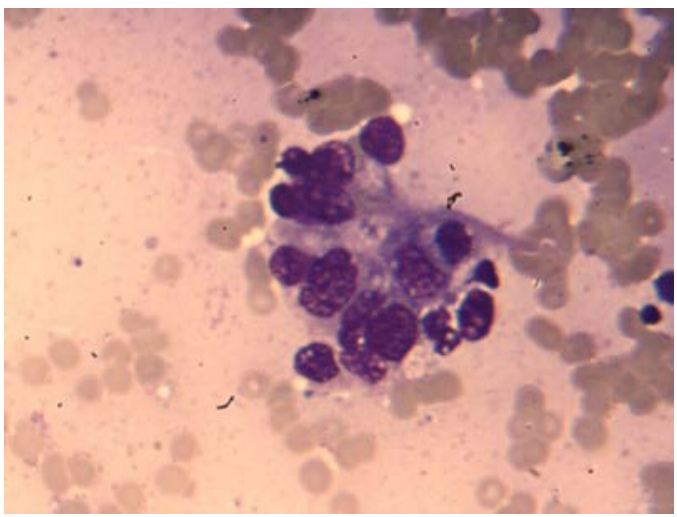

Figure 7. A different imagination of the same bone marrow examination of the ilium that reveals abnormal cells accompanied by mucous (stain $\mathrm{X}$ 400)

\section{Conflict of Interest}

The authors declare that there is no conflict of interest.

\section{References}

[1] Naito M, Yoshida Y, Aisu N, Tanimura S, Hoshino S, Tanaka T, Nimura S, Tamura K, Yamashita Y. A report of disseminated carcinomatosis of the bone marrow originating from transverse colon cancer successfully treated with chemotherapy using XELOX plus bevacizumab. Case Rep Oncol 2014; 7: 426-434.

[2] Takeda H, Nishikawa H, Tsumura T, Sekikawa A, Maruo T, Okabe Y, Kimura T, Wakasa, Osaki Y. Prominent hypereosinophilia with disseminated intravascular coagulation as an unusual presentation of advanced gastric cancer. Intern Med 53: 563-569, 2014.

[3] Yee Chao Y, Teng HC, Hung HC, King KC, Li CP, Chi KH, Sang-Hue Yen SH, Chang FY. Successful initial treatment with weekly etoposide, epirubicin, cisplatin, 5-Fluorouracil and leucovorin chemotherapy in advanced gastric cancer patients with disseminated intravascular coagulation. Jpn J Clin Oncol (2000) 30 (3): 122-125.

[4] Suzuki H, Matsuoka N, Ushimaru Y, Kakimoto Y, Toyoda A, Endo Y, Murakami O, et al. A case of disseminated carcinomatosis of the bone marrow with disseminated intravascular coagulation caused by advanced colon cancer successfully treated with SOX/bevacizumab. Gan To Kagaku Ryoho 2014 Aug; 41 (8): 1013-6.

[5] Ganno H, Nagahama T, Fukuda A, Takeuchi S, Fujiya K, Amagasa H, Kamikozuru $\mathrm{H}$, et al. A case of gastric cancer accompanied by disseminated carcinomatosis of the bone marrow successfully controlled by S-1 and cisplatin combination therapy. Gan To Kagaku Ryoho 2013 Nov; 40 (12): 2241-3.

[6] Isozaki Y, Yamanishi M, Utsunomiya S, Yamaguchi S, Okita M, Matsumoto N, Nagao Y, et al. A case of disseminated carcinomatosis of bone marrow with disseminated intravascular coagulation caused by advanced colon cancer treated by mFOLFOX6. Gan To Kagaku Ryoho 2011 Oct; 38 (10): 1705-8.

[7] Yamazaki K, Katsumura N, Ozawa N, Ogiso H, Hanai Y, Otsuji K, Toda K, et al. A case of mucinous adenocarcinoma of the sigmoid colon with disseminated carcinomatosis of the bone marrow successfully treated with FOLFOX4/bevacizumab. Gan To Kagaku Ryoho 2013 Aug; 40 (8): 1105-9.

[8] Higashiyama A, Kudo M, Nagasako T, Kawamura N, Abiko S, Yamamoto Y, Takano M, et al. Successful chemotherapy of carcinomatosis of the bone marrow with disseminated intravascular coagulation from a rectal carcinoma found by eosinophilia. Nihon Shokakibyo Gakkai Zasshi 2011 Jul; 108 (7): 1244-51.

[9] Yamauchi M, Okamoto Y, Doi M, Shinokazi K. mFOLFOX6 for treatment of anal canal cancer with disseminated carcinomatosis of bone marrow-a case report. To Kagaku Ryoho 2010 Nov; 37 (11): 2209-11.

[10] Ferrand FR, Gontier E, Guymar S, Fagot T, Ceccaldi B, Malfuson JV, de Revel T. Effectiveness and safe use of modified FOLFOX6 for metastatic gastric cancer with signet ring cell components complicated by disseminated intravascular coagulation and diffuse bone marrow carcinomatosis. Onkologie 2012; 35 (3): 118-20.

[11] Mizota A, Shitara K, Kondo C, Nomura M, Yokota T, Takahari D, Ura T, Muro K. A case of heavily pretreated rectal cancer with disseminated intravascular coagulation that improved following reintroduction of FOLFOX plus bevacizumab. Int J Clin Oncol 2011 Dec; 16 (6): 766-9.

[12] de Gramont A, Van Cutsem E, Schmoll HJ, Tabernero J, Clarke S, Moore MJ, Cunningham D, et al. Bevacizumab plus oxaliplatinbased chemotherapy as adjuvant treatment for colon cancer (AVANT): a phase 3 randomized controlled trial. Lancet Oncol 2012 Dec; 13 (12): 1225-33.

[13] Yoshida Y, Hoshino S, Shiwaku H, Beppu R, Tanimura S, Tanaka S, Yamashita Y. Early start of chemotherapy after resection of primary colon cancer with synchronous multiple liver metastases: a case report. Case Rep Oncol 2011; 4: 250-254. 